\title{
AN ABSTRACT SEMILINEAR VOLTERRA INTEGRODIFFERENTIAL EQUATION'
}

\author{
G. F. WEBB
}

ABSTRACT. The abstract semilinear Volterra integrodifferential equation

$$
u^{\prime}(t)=A u(t)+\int_{0}^{t} g(t-s, u(s)) d s+f(t), \quad t>0, u(0)=x \in X,
$$

is investigated, where $A$ is the infinitesimal generator of a semigroup of linear operators in a Banach space $X$ and $g$ is nonlinear and unbounded in its second place. Some results are proved concerning local existence, global existence, continuous dependence upon initial values, and asymptotic stability. The method used treats the equation in the domain of $A$ with the graph norm employing results from linear semigroup theory concerning abstract inhomogeneous linear differential equations.

1. Introduction. The purpose of this paper is to study the abstract semilinear Volterra integrodifferential initial value problem

$$
u^{\prime}(t)=A u(t)+\int_{0}^{t} g(t-s, u(s)) d s+f(t), \quad t \geqslant 0, u(0)=x \in X .
$$

In equation (1.1) $A$ is the infinitesimal generator of a linear semigroup in the Banach space $X$ and $g$ is, in general, a nonlinear unbounded operator from $\mathbf{R}^{+} \times X$ to $X$.

The equation (1.1) has many physical applications and arises in such problems as heat flow in materials with memory [3], [4], [13]. As a model for (1.1) one can take

$$
\begin{aligned}
& w_{t}(x, t)=w_{x x}(x, t)+\int_{0}^{t} k\left(t-s, w_{x x}(x, s)\right) d s+h(x, t), \\
& \quad 0<x<1, t>0, \\
& w(0, t)=w(1, t)=0, \quad t>0, \quad w(x, 0)=w_{0}(x), \quad 0<x<1 .
\end{aligned}
$$

For this example we let $X=L^{2}(0,1 ; \mathbf{R}), A z=z^{\prime \prime}, D(A)=\left\{z \in X: z^{\prime \prime} \in\right.$ $X, z(0)=z(1)=0\}, k: \mathbf{R}^{+} \times \mathbf{R} \rightarrow \mathbf{R}$ such that $k$ is continuous, $k(s, x)$ is continuously differentiable in its first place, $k(s, x)$ and $k_{1}(s, x)$ are Lipschitz continuous in $x$ uniformly in $s, f: \mathbf{R}^{+} \rightarrow X$ with $f(t)(x)=h(x, t)$, and $g$ :

Received by the editors December 20, 1976 and, in revised form, April 11, 1977.

AMS (MOS) subject classifications (1970). Primary 45N05; Secondary 47D05.

Key words and phrases. Abstract Volterra integrodifferential equation, semigroup of bounded linear operators, infinitesimal generator, existence, uniqueness, asymptotic behavior.

'This research was supported in part by the National Science Foundation Grant NSF MCS $75-06332$ A01. 
$\mathbf{R}^{+} \times D(A) \rightarrow X$ with $g(s, z)=k\left(s, z^{\prime \prime}\right)$. Equation (1.1) then becomes the abstract formulation of (1.2).

In the last few years considerable progress has been achieved in the investigation of (1.1). Some important results concerning existence, uniqueness, and asymptotic behavior in this theory may be found in the works listed in our references. In most of these works $g(s, z)$ is written as $a(s) \sigma(z)$ with a monotonicity condition on $\sigma$, thus allowing the nonlinear term to contribute to the existence of solutions. In our work the nonlinear term is treated as a perturbation of the linear equation. Our assumptions on the nonlinear term (which require a Lipschitz condition with respect to $A$ rather than monotonicity), then allow it to be overcome by the linear part of the equation. Our treatment permits the more general form of $g(s, z)$ and is valid in general Banach spaces. Our approach is essentially an extension from the linear to the nonlinear case of the methods of [7], [8], and [15].

2. The results. In what follows we let $X$ be a general Banach space with norm $\|$ and $A$ be the infinitesimal generator of a strongly continuous semigroup of bounded linear operators $T(t), t \geqslant 0$ in $X$ satisfying $|T(t)| \leqslant$ $e^{\omega t}, t \geqslant 0$, where $\omega$ is a real constant. We let $[D(A)]$ denote the Banach space which is the domain of $A$ with graph norm

$$
|x|_{A}=|x|+|A x|, \quad x \in D(A) .
$$

We make the following assumptions on $f$ and $g$ :

(2.1) $f: \mathbf{R}^{+} \rightarrow X$, where $f$ is continuously differentiable;

(2.2) $g: \mathbf{R}^{+} \times D \rightarrow X$, where $D$ is an open subset of $[D(A)], g$ is continuous, $g$ is continuously differentiable with respect to its first place, and for each $x \in D$ there exist a neighborhood $D_{x}$ about $x$ and continuous functions $b: \mathbf{R}^{+} \rightarrow \mathbf{R}^{+}$and $c: \mathbf{R}^{+} \rightarrow \mathbf{R}^{+}$such that

$$
\left|g\left(s, x_{1}\right)-g\left(s, x_{2}\right)\right| \leqslant b(s)\left|x_{1}-x_{2}\right|_{A}
$$

and

$$
\left|g_{1}\left(s, x_{1}\right)-g_{1}\left(s, x_{2}\right)\right| \leqslant c(s)\left|x_{1}-x_{2}\right|_{A}
$$

for all $s \in \mathbf{R}^{+}, x_{1}, x_{2} \in D_{x}$.

We require the following lemma, which is proved in [9, p. 486]:

LEMMA 2.1. Let $k:\left[0, t_{1}\right] \rightarrow X$ such that $k$ is continuously differentiable. If for $0 \leqslant t \leqslant t_{1}$,

$$
q(t) \stackrel{\text { def }}{=} \int_{0}^{t} T(t-s) k(s) d s
$$

then $q(t) \in D(A), q$ is continuously differentiable, and

$$
q^{\prime}(t)=A q(t)+k(t)=\int_{0}^{t} T(t-s) k^{\prime}(s) d s+T(t) k(0) .
$$

TheOrem 2.1. Let (2.1) and (2.2) hold. For each $x \in D$ there exists $t_{1}>0$ and $a$ unique function $u:\left[0, t_{1}\right] \rightarrow X$ such that $u$ is continuous from $\left[0, t_{1}\right]$ to $[D(A)], u$ is differentiable from $\left[0, t_{1}\right]$ to $X$, and $u$ satisfies (1.1). Furthermore, if 
$D=D_{x}=[D(A)]$, then the solution $u$ of $(1.1)$ exists on $\mathbf{R}^{+}$.

Proof. Let $x \in D$ and let $N$ be a neighborhood about $x$ in $[D(A)]$ such that $\bar{N} \subset D_{x}$. Let $t_{1}>0$ (we will specify $t_{1}$ later) and let $C=C\left(0, t_{1} ; \bar{N}\right)$. Define the mapping $K$ on $C$ by

$$
\begin{aligned}
(K u)(t)= & T(t) x+\int_{0}^{t} T(t-s) \int_{0}^{s} g(s-r, u(r)) d r d s \\
& +\int_{0}^{t} T(t-s) f(s) d s, \quad u \in C, 0<t \leqslant t_{1} .
\end{aligned}
$$

By virtue of the hypothesis we have placed on $g$, the function

$$
k(s)=\int_{0}^{s} g(s-r, u(r)) d r, \quad 0 \leqslant s \leqslant t_{1},
$$

is continuously differentiable from $\left[0, t_{1}\right]$ to $X$ and

$$
k^{\prime}(s)=g(0, u(s))+\int_{0}^{s} g_{1}(s-r, u(r)) d r .
$$

By Lemma 2.1, for $u \in C, 0 \leqslant t \leqslant t_{1},(K u)(t) \in D(A)$, and

$$
\begin{aligned}
(A K u)(t)= & T(t) A x+\int_{0}^{t} T(t-s)\left[g(0, u(s))+\int_{0}^{s} g_{1}(s-r, u(r)) d r\right] d s \\
& -\int_{0}^{t} g(t-s, u(s)) d s+\int_{0}^{t} T(t-s) f^{\prime}(s) d s \\
& +T(t) f(0)-f(t) .
\end{aligned}
$$

Thus, for $u \in C, K u$ and $A K u$ are both continuous from $\left[0, t_{1}\right]$ to $X$. If $t_{1}$ is chosen sufficiently small, then $K$ maps $C$ into $C$. Further, if $t_{1}$ is chosen sufficiently small, then $K$ is a contraction on $C$, since

$$
\begin{gathered}
|(K u)(t)-(K v)(t)| \leqslant \int_{0}^{t} e^{\omega(t-s)} \int_{0}^{s} b(s-r)|u(r)-v(r)|_{A} d r d s, \\
|(A K u)(t)-(A K v)(t)| \\
\leqslant \int_{0}^{t} e^{\omega(t-s)}\left[b(0)|u(s)-v(s)|_{A}+\int_{0}^{s} c(s-r)|u(r)-v(r)|_{A} d r\right] d s \\
\quad+\int_{0}^{t} b(t-s)|u(s)-v(s)|_{A} d s .
\end{gathered}
$$

By the Contraction Mapping Theorem there exists a unique $u \in C$ such that $K u=u$. From (2.3) and Lemma 2.1 we see that $u:\left[0, t_{1}\right] \rightarrow[D(A)]$ is continuous, $u:\left[0, t_{1}\right] \rightarrow X$ is differentiable, and $u$ satisfies (1.1).

Finally, if $D=D_{x}=[D(A)]$, then $N$ can be chosen as $[D(A)]$. In this case $t_{1}$ does not depend on $x$ nor on $f(t)$ and the solution $u$ can be continued to $+\infty$. That is, let $\hat{x}=u\left(t_{1}\right)$ and

$$
\hat{f}(t)=f(t)+\int_{0}^{t_{1}} g\left(t+t_{1}-s, u(s)\right) d s .
$$

Then, $\hat{x} \in D(A)$ and $\hat{f}$ is continuously differentiable from $\mathbf{R}^{+}$to $X$. By the 
argument above there exists a unique function $\hat{u}:\left[0, t_{1}\right] \rightarrow X$ such that $\hat{u}$ is continuous from $\left[0, t_{1}\right]$ to $[D(A)], \hat{u}$ is differentiable from $\left[0, t_{1}\right]$ to $X$, and

$$
\hat{u}^{\prime}(t)=A \hat{u}(t)+\int_{0}^{t} g(t-s, \hat{u}(s)) d s+\hat{f}(t), \quad 0 \leqslant t \leqslant t_{1}, \hat{u}(0)=\hat{x} .
$$

Define $u\left(t+t_{1}\right)=\hat{u}(t), 0 \leqslant t \leqslant t_{1}$, and

$$
\begin{aligned}
u^{\prime}\left(t+t_{1}\right) & =A u\left(t+t_{1}\right)+\int_{0}^{t} g\left(t-s, u\left(s+t_{1}\right)\right) d s+\hat{f}(t) \\
& =A u\left(t+t_{1}\right)+\int_{0}^{t+t_{1}} g\left(t+t_{1}-s, u(s)\right) d s+f(t) .
\end{aligned}
$$

Repeating this process, we see $u$ exists on $\mathbf{R}^{+}$.

Theorem 2.2. Let (2.1) and (2.2) hold and let $x \in D$. There exist continuous functions $\alpha: \mathbf{R}^{+} \rightarrow \mathbf{R}^{+}$and $\beta: \mathbf{R}^{+} \rightarrow \mathbf{R}^{+}$such that if $u_{1}$ and $u_{2}$ satisfy (1.1) for $0 \leqslant t \leqslant t_{1}$ with $u_{1}(0)=x_{1}$ and $u_{2}(0)=x_{2}, u_{1}(t)$ and $u_{2}(t) \in D_{x}$ for $0 \leqslant t \leqslant$ $t_{1}$, then

$$
\begin{aligned}
\left|u_{1}(t)-u_{2}(t)\right|_{A} \leqslant\left|x_{1}-x_{2}\right|_{A} \exp ((\alpha(t)+\beta(t)+b(0)+\omega) t), & 0 \leqslant t \leqslant t_{1} .
\end{aligned}
$$

Proof. Define $\alpha(t)=\int_{0}^{t} e^{-\omega s}(b(s)+c(s)) d s, t \geqslant 0$, and

$$
\beta(t)=\max _{0 \leqslant s \leqslant t} e^{-\omega s} b(s), \quad t \geqslant 0 .
$$

Let $p_{1}(t)=e^{-\omega t}\left|u_{1}(t)-u_{2}(t)\right|, \quad p_{2}(t)=e^{-\omega t}\left|A\left(u_{1}(t)-u_{2}(t)\right)\right|$, and $p(t)=$ $p_{1}(t)+p_{2}(t), 0 \leqslant t \leqslant t_{1}$. Using (2.3) and (2.4) we have

$$
\begin{aligned}
& p_{1}(t) \leqslant\left|x_{1}-x_{2}\right|+\int_{0}^{t} e^{-\omega s} \int_{0}^{s} b(s-r) e^{\omega r} p(r) d r d s \\
& \begin{aligned}
p_{2}(t) \leqslant & \left|A\left(x_{1}-x_{2}\right)\right|+\int_{0}^{t} b(0) p(s) d s \\
& +\int_{0}^{t} e^{-\omega s} \int_{0}^{s} c(s-r) e^{\omega r} p(r) d r d s+\int_{0}^{t} e^{-\omega(t-s)} b(t-s) p(s) d s \\
p(t) \leqslant & \left|x_{1}-x_{2}\right|_{A}+\int_{0}^{t} \int_{0}^{t-r} e^{-\omega s}(b(s)+c(s)) p(r) d s d r \\
& +b(0) \int_{0}^{t} p(s) d s+\int_{0}^{t} e^{-\omega(t-s)} b(t-s) p(s) d s \\
\leqslant & \left|x_{1}-x_{2}\right|_{A}+(\alpha(t)+\beta(t)+b(0)) \int_{0}^{t} p(s) d s
\end{aligned}
\end{aligned}
$$

An application of Gronwall's Lemma now yields (2.5).

Corollary 2.1. Let (2.1) and (2.2) hold with $D=D_{x}=[D(A)]$ and suppose there exist constants $\alpha_{0}$ and $\beta_{0}$ such that $\int_{0}^{t} e^{-\omega s}(b(s)+c(s)) d s \leqslant \alpha_{0}$ for $t \geqslant 0$, $e^{-\omega t} b(t) \leqslant \beta_{0}$ for $t \geqslant 0$, and $\alpha_{0}+\beta_{0}+b(0)+\omega={ }^{\text {def }} \gamma \leqslant 0$. Then the solutions of (1.1) are exponentially asymptotically stable in the A-norm in the following sense: if $u_{1}(t), u_{2}(t)$ are the solutions of $(1.1)$ for $u_{1}(0)=x_{1}, u_{2}(0)=$ 
$x_{2}$, respectively, then $\left|u_{1}(t)-u_{2}(t)\right|_{A} \leqslant\left|x_{1}-x_{2}\right|_{A} e^{\gamma t}, t>0$.

Proof. The proof follows from Theorem 2.2 by observing that $\alpha(t)$ and $\beta(t)$ satisfy $\alpha(t) \leqslant \alpha_{0}$ and $\beta(t) \leqslant \beta_{0}$.

THEOREM 2.3. Let (2.1) and (2.2) hold and, in addition, let $g(t, x)$ and $g_{1}(t, x)$ be Lipschitz continuous on bounded sets of $x$ uniformly in finite intervals of $t$. If $x \in D$ and $u$ is a noncontinuable solution of $(1.1)$ on $[0, d)$, then either $d=+\infty$ or given any closed bounded set $U$ in $D$ there exists a sequence $t_{k} \rightarrow d^{-}$such that $u\left(t_{k}\right) \notin U$.

Proof. Assume $d<\infty$ and the conclusion of the theorem is false. Then there exists a closed bounded set $U$ in $D$ such that $u(t) \in U$ for $0<t<d$. For $0<t<t+h<d$, (2.3) implies

$$
\begin{aligned}
|u(t+h)-u(t)| \leqslant|T(t+h) x-T(t) x| \\
+\left|\int_{-h}^{0} T(t-s) \int_{0}^{s+h} g(s+h-r, u(r)) d r d s\right| \\
+\left|\int_{0}^{t} T(t-s)\left(\int_{0}^{s+h} g(s+h-r, u(r)) d r-\int_{0}^{s} g(s-r, u(r)) d r\right) d s\right| \\
+\left|\int_{-h}^{0} T(t-s) f(s+h) d s\right|+\left|\int_{0}^{t} T(t-s)(f(s+h)-f(s)) d s\right| .
\end{aligned}
$$

Using the estimate below, where $b$ is the uniform Lipschitz constant of $g(t, \cdot)$ on $[0, d] \times u$,

$$
\begin{aligned}
& \left|\int_{0}^{t} T(t-s)\left(\int_{0}^{s+h} g(s+h-r, u(r)) d r-\int_{0}^{s} g(s-r, u(r)) d r\right) d s\right| \\
& =\mid \int_{0}^{t} T(t-s)\left(\int_{-h}^{0} g(s-r, u(r+h)) d r\right. \\
& \left.\quad+\int_{0}^{s}(g(s-r, u(r+h))-g(s-r, u(r))) d r\right) d s \mid \\
& \leqslant\left|\int_{0}^{t} T(t-s) \int_{-h}^{0} g(s-r, u(r+h)) d r d s\right| \\
& \quad+\int_{0}^{t} \int_{r}^{t}|T(t-s)| b|u(r+h)-u(r)|_{A} d s d r,
\end{aligned}
$$

we see that there exist constants $L$ and $M$ such that for $0<t<t+h<d$,

$$
|u(t+h)-u(t)| \leqslant L h+M \int_{0}^{t}|u(s+h)-u(s)|_{A} d s .
$$

In a similar fashion we use (2.4) to show that for $0<t<t+h<d$,

$$
|A(u(t+h)-u(t))| \leqslant \hat{L}(h)+\hat{M} \int_{0}^{t}|u(s+h)-u(s)|_{A} d s
$$


for some continuous function $\hat{L}: \mathbf{R}^{+} \rightarrow \mathbf{R}^{+}$with $\hat{L}(0)=0$ and some constant $\hat{M}$. Using (2.6), (2.7), and Gronwall's Lemma, we have for $0<t<t+h<$ $d,|u(t+h)-u(t)|_{A} \leqslant(L+\hat{L})(h) \exp ((M+\hat{M}) t)$. Thus, $\lim _{t \rightarrow d^{-}} u(t)$ exists in $[D(A)]$ and is in $D$. By Theorem $2.1, u(t)$ can be continued past $d$, contradicting the noncontinuability hypothesis.

Corollary 2.2. Let (2.1) and (2.2) hold, let $D=[D(A)]$, and let $g(t, x)$ and $g_{1}(t, x)$ be Lipschitz continuous on bounded sets of $x$ in $D$ uniformly in finite intervals of $t$. If $x \in D$ and $u$ is a noncontinuable solution of $(1.1)$ on $[0, d)$, then either $d=+\infty$ or $\varlimsup_{\lim _{t \rightarrow d^{-}}}|u(t)|_{A}=+\infty$.

Proof. Suppose $\varlimsup_{t \rightarrow d^{-}}|u(t)|_{A}<\infty$. Let $U$ be the closure in $[D(A)]$ of $\{u(t): 0 \leqslant t<d\}$. Then $U$ is closed and bounded, $U \subset D$, and by Theorem 2.3, we must have $d=+\infty$.

We conclude with the observation that the hypothesis $g:[D(A)] \rightarrow X$ such that $g$ is Lipschitz continuous is not sufficient to guarantee the existence of a solution to

$$
u^{\prime}(t)=A u(t)+g(u(t)), \quad t \geqslant 0, u(0)=x \in D(A)
$$

(for example, take $g=-2 A$ ).

\section{REFERENCES}

1. V. Barbu, Nonlinear Volterra equations in a Hilbert space, SIAM J. Math. Anal. 6 (1975), 728-741.

2. Nonlinear semigroups and differential equations in Banach spaces, Noordhoff, Leyden, 1976.

3. B. D. Coleman and M. E. Gurtin, Equi presence and constitutive equations for rigid heat conductors, Z. Angew. Math. Phys. 18 (1967), 199-208.

4. B. D. Coleman and V. J. Mizel, Norms and semigroups in the theory of fading memory, Arch. Rational Mech. Anal. 28 (1966), 87-123.

5. M. G. Crandall, S.-O. Londen and J. A. Nohel, An abstract nonlinear Volterra integrodifferential equation, MRC Technical Summary Report \#1684, University of Wisconsin, Madison, 1976.

6. C. M. Dafermos, An abstract Volterra equation with applications to linear viscoelasticity, J. Differential Equations 7 (1970), 554-569.

7. A. Friedman, Monotonicity of solutions of Volterra integral equations in Banach space, Trans. Amer. Math. Soc. 138 (1969), 129-148.

8. A. Friedman and M. Shinbrot, Volterra integral equations in Banach space, Trans. Amer. Math. Soc. 126 (1967), 131-179.

9. T. Kato, Perturbation theory for linear operators, Springer-Verlag, New York, 1966.

10. S.-O. Londen, An existence result on a Volterra equation in a Banach space, Trans. Amer. Math. Soc. (to appear).

11. __ On an integral equation in a Hilbert space, SIAM J. Math. Anal. (to appear).

12. R. C. MacCamy, Stability theorems for a class of functional differential equations, SIAM J. Math. Anal. (to appear).

13. An integro-differential equation with applications in heat flow (to appear).

14. R. C. MacCamy and J. S. W. Wong, Stability theorems for some functional differential equations, Trans. Amer. Math. Soc. 164 (1972), 1-37.

15. R. K. Miller, Volterra integral equations in a Banach space, Funkcial. Ekvac. 18 (1975), 163-194.

16. M. Slemrod, $A$ hereditary partial differential equation with applications in the theory of simple fluids, Arch. Rational Mech. Anal. (to appear).

Department of Mathematics, Vanderbilt University, Nashville, Tennessee 37235 